\title{
Financial toxicity in sarcoma patients and survivors in Germany: results from the multicenter PROSa study
}

\author{
Matthias Büttner ${ }^{1,2} \cdot$ Susanne Singer ${ }^{1,2} \cdot$ Leopold Hentschel $^{3} \cdot$ Stephan Richter ${ }^{4} \cdot$ Peter Hohenberger $^{5}$. \\ Bernd Kasper $^{6} \cdot$ Dimosthenis Andreou $^{7,8} \cdot$ Daniel Pink $^{8,9} \cdot$ Kathy Taylor $^{1,2} \cdot$ Karin Arndt $^{10} \cdot$ Martin Bornhäuser $^{3,4}$. \\ Jochen Schmitt ${ }^{5,11} \cdot$ Markus K. Schuler ${ }^{4} \cdot$ Martin Eichler ${ }^{3,4}$
}

Received: 9 March 2021 / Accepted: 26 June 2021 / Published online: 11 July 2021

(c) The Author(s) 2021

\begin{abstract}
Purpose Cancer patients have been shown to frequently suffer from financial burden before, during, and after treatment. However, the financial toxicity of patients with sarcoma has seldom been assessed. Therefore, the aim of this study was to evaluate whether financial toxicity is a problem for sarcoma patients in Germany and identify associated risk factors.

Methods Patients for this analysis were obtained from a multicenter prospective cohort study conducted in Germany. Using the financial difficulties scale of the EORTC QLQ-C30, financial toxicity was considered to be present if the score exceeded a pre-defined threshold for clinical importance. Comparisons to an age- and sex-matched norm population were performed. A multivariate logistic regression using stepwise backward selection was used to identify factors associated with financial toxicity.

Results We included 1103 sarcoma patients treated in 39 centers and clinics; 498 (44.7\%) patients reported financial toxicity. Sarcoma patients had 2.5 times the odds of reporting financial difficulties compared to an age- and sex-matched norm population. Patient age $<40$ and $>52.5$ years, higher education status, higher income, and disease progression (compared to patients with complete remission) were associated with lower odds of reporting financial toxicity. Receiving a disability pension, being currently on sick leave, and having a disability pass were statistically significantly associated with higher odds of reporting financial toxicity.

Conclusion Financial toxicity is present in about half of German sarcoma patients, making it a relevant quality of life topic for patients and decision-makers.
\end{abstract}

Keywords Sarcoma $\cdot$ Financial toxicity $\cdot$ Burden $\cdot$ Cancer $\cdot$ Germany $\cdot$ Financial difficulties

Matthias Büttner

matbuett@uni-mainz.de

1 Division of Epidemiology and Health Services Research, Institute of Medical Biostatistics, Epidemiology and Informatics (IMBEI), University Medical Center Mainz, Obere Zahlbacher Straße 69, 55131 Mainz, Germany

2 University Cancer Centre, Mainz, Germany

3 National Center for Tumor Diseases (NCT/UCC), Dresden, Germany

4 Clinic and Polyclinic for Internal Medicine I, University Hospital Carl Gustav Carus, TU Dresden, Dresden, Germany

5 Division of Surgical Oncology \& Thoracic Surgery, Mannheim University Medical Center, University of Heidelberg, Mannheim, Germany
6 Sarcoma Unit, Interdisciplinary Tumor Center, University Medical Center Mannheim, Mannheim, Germany

7 Department of General Orthopedics and Tumor Orthopedics, University Hospital Munster, Münster, Germany

8 Sarcoma Center Berlin-Brandenburg, Helios Hospital Bad Saarow, Bad Saarow, Germany

9 Department of Internal Medicine C, University Hospital Greifswald, Greifswald, Germany

10 German Sarcoma Foundation, Woelfersheim, Germany

11 Center for Evidence-Based Healthcare, University Hospital Carl Gustav Carus, Technical University Dresden, Dresden, Germany 


\section{Introduction}

Sarcomas are a rare group of cancers of mesenchymal origin and are estimated to represent 1 to $2 \%$ of adult cancers worldwide [1]. The 5 -year overall survival probability amounts to approximately $60 \%$ and depends on a variety of risk factors [2]. Quality of life impairments among sarcoma patients have been reported across all sarcoma subgroups and are present during diagnosis, treatment, and follow-up [3].

One important aspect of quality of life that has gained increasing awareness within the last years is the financial burden of cancer patients. The financial burden of cancer refers not only to out-of-pocket payments (OOPPs) and income losses, but also material consequences and psychological effects or behavioral changes that are then expressed as financial toxicity [4-7]. It has been shown that patients reporting financial difficulties may have an increased risk of distress, anxiety, and depression [8, 9], show impairments in quality of life [8-11], and may even have worse survival [12]. This problem is not only observed in countries with high OOPPs, like the USA, but also in countries with universal health coverage [13-16].

Financial toxicity studies are often conducted in general cancer populations or are performed for the most common cancers, such as lung, breast, or colon cancer [13-19]. To our knowledge, studies evaluating financial toxicity in sarcoma patients in detail have not been performed. It has, however, been shown that the financial impact of the disease and the resulting financial toxicity is an important and relevant topic for patients with sarcoma [17]. Additionally, patients can be diagnosed with sarcoma throughout the whole course of life $[1,2]$ and therefore also during their social, educational, and occupational development and this might also influence their financial abilities throughout the rest of their life.

Therefore, the aim of this study was to evaluate the extent of financial toxicity in German sarcoma patients and survivors in Germany and identify possible risk factors.

\section{Methods}

\section{Design}

Patients for this analysis were obtained from the PROSa study, which is a multicenter prospective cohort study conducted in Germany with two follow-ups at 6 and 12 months after baseline. Follow-up was defined as a visit to one of the participating study centers [18]. The baseline data used for this analysis were obtained between 09/2017 and 02/2019. Inclusion criteria for the PROSa study were a confirmed diagnosis of any type of sarcoma regardless of the current stage of the disease course (at diagnosis, in treatment, or in follow-up) and the ability to give written informed consent. Patients who were not able to fill out questionnaires due to linguistic reasons (restricted to German language) or cognitive impairment were excluded from the study. Ethical approval was obtained by the ethics committee of the Technical University of Dresden (AZ: EK 1,790,422,017) and the ethics committees of the participating centers. A more detailed description of the PROSa study can be found in [18].

\section{Assessments}

Clinical data of the participants were obtained using case report forms through the participating medical centers. Aftercare was defined as "yes" for patients with being in after care or for having completed their treatment. Sociodemographic data and patient reported outcomes (PROs) were directly obtained from the patient by letter or online questionnaires. The following socioeconomic and sociodemographic variables were used for the analysis: age, sex, education, occupation, employment status, income, sick leave status, and whether a patient had a disability pass. Education was defined by the highest educational certificate attained divided into the following categories: none to secondary ( $8 / 9$ years), secondary school (10 years), vocational baccalaureate, high school/baccalaureate, and other/ unknown. Occupation was assessed by the classification of labor type (e.g., blue-collar worker, civil servant) and employment status was determined by the employment setting (e.g., self-employed, retired, etc.). Income was assessed using the OECD equivalence scale [19] as a continuous variable but also categorized into income groups $\leq € 1250, € 1251$ to $€ 1750, € 1751$ to $€ 2250, € 2251$ to $€ 2750,>€ 2750$, and unknown.

For the assessment of financial toxicity, the financial difficulties scale of the EORTC QLQ-C30 was used [20]. This 1-item scale measures whether the physical conditions or the medical treatment has caused any financial difficulties within the last week using a four-point Likert scale ("not at all," "a little," "quite a bit," and "very much"). The answer is converted to a scale ranging from 0 to 100 , with high values indicating a high financial burden perceived by the patient in accordance with the scoring manual of the EORTC [21]. Patients were considered to have financial toxicity if their score in the financial difficulties scale was above the threshold for clinical importance (17 points) identified by Giesinger et al. [22]. Patients with a score above 17 points at least report any financial difficulties ("a little," "quite a bit," or "very much"). 


\section{Statistical analysis}

The analysis was performed on a cross-sectional basis. Patient characteristics are expressed as mean values or percentages according to the data. Univariate comparisons between patients with financial toxicity and patients without were conducted using Chi-square tests. Financial difficulty scores of all sarcoma patients, curative sarcoma patients, and palliative sarcoma patients were compared to a norm population [23]. Multivariate logistic regression with backward stepwise selection with $p>0.1$ as the exclusion criterion was performed with reporting financial difficulties (yes/no) as the dependent variable and clinical and socioeconomic characteristics as independent variables (Table 1). Variables were included in regression after being tested for non-multicollinearity (correlation $<0.8$ ). All statistical analyses were performed using IBM SPSS Statistics version 25.0 (Armonk, NY).

\section{Results}

\section{Sample and patient characteristics}

In total, 1309 sarcoma patients from 39 sarcoma centers and clinics participated in the study, of whom 1103 (84.3\%) provided data regarding financial toxicity. The mean age of the study population at recruitment was 52.6 years, and $48.7 \%$ of the participants were female. Further sociodemographic and clinical characteristics can be found in Table 1 .

\section{Financial toxicity in German sarcoma patients}

A total of 498 (44.7\%) of the sarcoma patients reported suffering from financial difficulties above the threshold for clinical importance. Differences between patients with and without financial toxicity are presented in Table 1 . Statistically significant differences between the two groups were seen for age, education, occupation, income, employment, disability pass, biological behavior of the tumor, metastasis at baseline, aftercare status, and chemotherapy.

Compared to the German norm population [23], sarcoma patients in the study had statistically higher scores, indicating more problems in the financial difficulties scale; this difference also persisted after stratification into curative and palliative sarcoma patients (Fig. 1). All sarcoma patients had 2.5 times the odds of reporting financial difficulties compared to the age- and sex-matched norm population.

\section{Variables associated with financial toxicity in German sarcoma patients}

The final variable selection of the multivariate logistic regression with backward stepwise selection and the respective results can be found in Table 2. Compared to the age group from 40 to $<52.5$ years, younger $(18$ to $<27.5$ years (OR: $0.45 ; 95 \%$ CI $[0.23 ; 0.88]$ ), 27.5 to $<40$ years (OR: $0.54 ; 95 \%$ CI $[0.31 ; 0.94]))$ and older $(52.5$ to $<65$ years (OR: $0.66 ; 95 \%$ CI $[0.44 ; 0.99]), 65$ to $<77.5$ years (OR: $0.25 ; 95 \%$ CI $[0.12 ; 0.49])$, and $>77.5$ years (OR: $0.27 ; 95 \%$ CI $[0.12 ; 0.62]))$ age groups had statistically lower odds of reporting financial toxicity. Higher education (compared to none to secondary school; secondary school (OR: $0.57 ; 95 \%$ CI $[0.39 ; 0.84]$ ), vocational baccalaureate (OR: $0.67 ; 95 \%$ CI $[0.39 ; 1.15]$ ), high school/baccalaureate (OR: $0.51 ; 95 \%$ CI $[0.33 ; 0.79])$ ) and higher income (compared to $\leq € 1250$; $€ 1250.1$ to $€ 1750$ (OR: 0.93 ; 95\% CI [0.60;1.43]), $€ 1750.1$ to $€ 2250$ (OR: $0.47 ; 95 \%$ CI [0.30;0.72]), €2250.1 to €2750 (OR: $0.24 ; 95 \%$ CI [0.13;0.45]), $\geq € 2750.1$ (OR0.33; 95\% CI $[0.12 ; 0.53]))$ were also associated with having lower chances of reporting financial toxicity. Receiving a disability pension (OR: $3.29 ; 95 \%$ CI [1.95;5.54], compared to employed/selfemployed), being currently on sick leave (OR: $2.40 ; 95 \%$ CI [1.60;3.62]), compared to not being currently on sick leave), and having a disability pass (OR: 2.52; 95\% CI [1.81;3.49]) were statistically significantly associated with higher odds of reporting financial toxicity. Patients more than 5 years post diagnosis (compared to 0 to $<0.5$ years since diagnosis) reported statistically significant lower odds (OR: $0.58 ; 95 \%$ CI $[0.35 ; 0.94])$ of reporting financial toxicity. Compared to patients with complete remission, patients with progressive disease had statistically significant lower odds (OR: 0.48; $95 \%$ CI [0.30;0.77]) of reporting financial toxicity, while for patients with partial remission/stable disease no differences in odds (OR: 0.96; 95\% CI [0.67;1.37]) were observed.

\section{Discussion}

Our analysis showed that sarcoma patients suffer from financial difficulties in a universal health coverage system like Germany's. $44.7 \%$ of the patients reported financial difficulties measured by the EORTC QLQ-C 30 , and 2.5 times the odds of reporting financial toxicity compared to an age- and sex matched norm population was observed.

These impairments regarding financial difficulties are in line with other studies using the financial difficulties scale of the QLQ-C30 in sarcoma patients [24-28]. Hudgens et al. [24] reported a financial difficulties score of 25.0 (SD: 29.5) at baseline and 29.5 (SD: 34.2 ) at progression in a study consisting of 452 sarcoma patients with advanced, progressing disease. A longitudinal study by Paredes et al. [25] with 36 sarcoma patients found higher financial difficulties scores compared to a norm population in the diagnostic phase and 4 months after initiation of treatment.

Financial difficulties are not restricted to sarcoma patients and have been observed in other cancer populations across different health care settings [13-16]. Financial toxicity 
Table 1 Description of baseline characteristics

\begin{tabular}{|c|c|c|c|c|}
\hline Variable & Value & $\begin{array}{l}\text { All } \\
\mathrm{N}=1103(\text { column } \%)\end{array}$ & $\begin{array}{l}\text { Financial toxicity-yes } \\
\mathrm{N}=498(44.7 \%)\end{array}$ & $\begin{array}{l}\text { Financial toxicity-no } \\
\mathrm{N}=605(54.9 \%)\end{array}$ \\
\hline \multicolumn{5}{|l|}{ Socioeconomic factors } \\
\hline \multirow[t]{2}{*}{ Sex } & Female & $537(48.7)$ & $243(48.8)$ & $294(48.7)$ \\
\hline & Male & $565(51.3)$ & $255(51.2)$ & $310(51.3)$ \\
\hline \multirow[t]{2}{*}{ Age at study inclusion $*, * *$} & Mean & $56.6(\mathrm{SD} 15.8)$ & $54.5(\mathrm{SD} 14.5)$ & $58.4(\mathrm{SD} 16.6)$ \\
\hline & Median IQR & $58.4(47.6 ; 68.5)$ & $55.4(46.6 ; 63.6)$ & $61.6(49.8 ; 70.6)$ \\
\hline \multirow{6}{*}{$\begin{array}{l}\text { Age at study inclusion- } \\
\text { group* }\end{array}$} & $18-<27.5$ years & $78(7.1)$ & $33(6.7)$ & $45(7.4)$ \\
\hline & $27.5-<40$ year & $104(9.4)$ & $44(8.9)$ & $60(9.9)$ \\
\hline & $40-<52.5$ years & $194(17.6)$ & $121(24.4)$ & $73(12.1)$ \\
\hline & $52.5-<65$ years & $366(33.2)$ & $194(39.1)$ & $172(28.4)$ \\
\hline & $65-<77.5$ years & $277(25.2)$ & $81(16.3)$ & $196(32.4)$ \\
\hline & $>77.5$ years & $82(7.4)$ & $23(4.6)$ & $59(9.8)$ \\
\hline \multirow[t]{3}{*}{ Household size (living alone)\# } & No & $887(80.4)$ & $400(80.3)$ & $487(80.5)$ \\
\hline & Yes & $206(18.7)$ & $93(18.7)$ & $113(18.7)$ \\
\hline & Unknown & $10(0.9)$ & $5(1.0)$ & $5(0.8)$ \\
\hline \multirow[t]{3}{*}{ Children in household\# } & No & $884(80.1)$ & $392(78.7)$ & $492(81.3)$ \\
\hline & Yes & $151(13.7)$ & $73(14.7)$ & $78(12.9)$ \\
\hline & Unknown & $68(6.2)$ & $33(6.6)$ & $35(5.8)$ \\
\hline \multirow[t]{5}{*}{ School leaving certificate*,\# } & $\begin{array}{l}\text { None to secondary school } \\
\text { (8/9 years) }\end{array}$ & $264(23.9)$ & $143(28.7)$ & $121(20.0)$ \\
\hline & Secondary school (10 years) & $376(34.1)$ & $177(35.5)$ & $199(32.9)$ \\
\hline & Vocational baccalaureate & $120(10.9)$ & $51(10.2)$ & $69(11.4)$ \\
\hline & High school/baccalaureate & 317 (28.7) & $115(23.1)$ & $202(33.4)$ \\
\hline & Something else/unknown & $26(2.4)$ & $12(2.4)$ & $14(2.3)$ \\
\hline \multirow[t]{5}{*}{ Occupational status*,\# } & Blue-collar worker & $208(18.9)$ & $118(23.7)$ & $90(14.9)$ \\
\hline & Civil servant (1) & $81(7.3)$ & $23(4.6)$ & $58(9.6)$ \\
\hline & White collar worker (2) & $611(55.4)$ & $266(53.4)$ & $345(57.0)$ \\
\hline & Self-employed (3) & $104(9.4)$ & $49(9.8)$ & $55(9.1)$ \\
\hline & Unknown/not applicable (4) & $99(9.0)$ & $42(8.4)$ & $57(9.4)$ \\
\hline Equivalent income*,** & Mean (standard deviation) & $2047 €(1105 €)$ & $1800 €(1010 €)$ & $2251 €(1138 €)$ \\
\hline \multirow[t]{6}{*}{ Equivalized income*,\# } & $\leq 1250$ & $231(20.9)$ & $135(27.1)$ & $96(15.9)$ \\
\hline & $1250.1-1750$ & $213(19.3)$ & $115(23.1)$ & $98(16.2)$ \\
\hline & $1750.1-2250$ & $247(22.4)$ & $103(20.7)$ & $144(23.8)$ \\
\hline & $2250.1-2750$ & $89(8.1)$ & $26(5.2)$ & $63(10.4)$ \\
\hline & $\geq 2750.1$ & $184(16.7)$ & $58(11.6)$ & $126(20.8)$ \\
\hline & Unknown & 139 (12.6) & $61(12.2)$ & $78(12.9)$ \\
\hline \multirow[t]{7}{*}{ Employment status*,\# } & Employed/self-employed & $490(44.4)$ & $226(45.4)$ & $264(43.6)$ \\
\hline & Unemployed & $45(4.1)$ & $31(6.2)$ & $14(2.3)$ \\
\hline & Disability pension & $138(12.5)$ & $103(20.7)$ & $35(5.8)$ \\
\hline & $\begin{array}{l}\text { Early retirement/retirement pen- } \\
\text { sion/partial retirement }\end{array}$ & $376(34.1)$ & $114(22.9)$ & $262(43.3)$ \\
\hline & Housewife/houseman & $26(2.4)$ & $11(2.2)$ & $15(2.5)$ \\
\hline & School, apprenticeship, study & $17(1.5)$ & $8(1.6)$ & $9(1.5)$ \\
\hline & Unknown & $11(1.0)$ & $5(1.0)$ & $6(1.0)$ \\
\hline \multirow[t]{3}{*}{ Sick leave*,\# } & No & $822(74.5)$ & $327(65.7)$ & $495(81.8)$ \\
\hline & Yes & $257(23.3)$ & $163(32.7)$ & $94(15.5)$ \\
\hline & Unknown & $24(2.2)$ & $8(1.6)$ & $16(2.6)$ \\
\hline \multirow[t]{3}{*}{ Disabled person pass*,\# } & No & $405(36.7)$ & $131(26.3)$ & $274(45.3)$ \\
\hline & Yes & $689(62.5)$ & $364(73.1)$ & $325(53.7)$ \\
\hline & Unknown & $9(0.8)$ & $3(0.6)$ & $6(1.0)$ \\
\hline
\end{tabular}


Table 1 (continued)

\begin{tabular}{|c|c|c|c|c|}
\hline Variable & Value & $\begin{array}{l}\text { All } \\
\mathrm{N}=1103(\text { column } \%)\end{array}$ & $\begin{array}{l}\text { Financial toxicity-yes } \\
\mathrm{N}=498(44.7 \%)\end{array}$ & $\begin{array}{l}\text { Financial toxicity-no } \\
\mathrm{N}=605(54.9 \%)\end{array}$ \\
\hline \multicolumn{5}{|l|}{ Clinical factors } \\
\hline \multirow[t]{5}{*}{ Time since diagnosis\# } & $0-<0.5$ years & $209(19.0)$ & $95(19.1)$ & $114(18.9)$ \\
\hline & $0.5-<1$ year & $126(11.4)$ & $67(13.5)$ & $59(9.8)$ \\
\hline & $1-<2$ years & $165(15.0)$ & $79(15.9)$ & $86(14.2)$ \\
\hline & $2-<5$ years & $290(26.3)$ & $131(26.4)$ & $159(26.3)$ \\
\hline & More than 5 years & $311(28.2)$ & $125(25.2)$ & $186(30.8)$ \\
\hline \multirow[t]{10}{*}{ Sarcoma type-detail§ } & Liposarcoma & $210(19.1)$ & $94(19.0)$ & $116(19.2)$ \\
\hline & Unclassified sarcoma (1) & $163(14.8)$ & $68(13.7)$ & $95(15.7)$ \\
\hline & $\begin{array}{l}\text { Fibroblastic, myofibroblastic, } \\
\text { fibrohistiocytic sarcoma (2) }\end{array}$ & $130(11.8)$ & $52(10.5)$ & $78(12.9)$ \\
\hline & GIST (3) & $130(11.8)$ & $51(10.3)$ & $79(13.0)$ \\
\hline & Leiomyosarcoma (4) & $131(11.9)$ & $66(13.3)$ & $65(10.8)$ \\
\hline & Osteosarcoma (5) & $71(6.5)$ & $30(6.0)$ & $41(6.8)$ \\
\hline & Chondrosarcoma (6) & $63(5.7)$ & $32(6.5)$ & $31(5.1)$ \\
\hline & Ewing sarcoma (7) & $43(3.9)$ & $28(5.6)$ & $15(2.5)$ \\
\hline & Synovial sarcoma (8) & $47(4.3)$ & $24(4.8)$ & $23(3.8)$ \\
\hline & Other (9) & $112(10.2)$ & $51(10.3)$ & $61(10.1)$ \\
\hline \multirow[t]{3}{*}{ Site $\S$} & Trunk & $518(47.1)$ & $231(44.6)$ & $287(47.5)$ \\
\hline & Limbs & $522(47.5)$ & $234(47.1)$ & $288(47.7)$ \\
\hline & Somewhere else & $60(5.5)$ & $31(6.3)$ & $29(4.8)$ \\
\hline \multirow[t]{3}{*}{ Grading§ } & Low grade & $137(12.4)$ & $52(10.4)$ & $85(14.0)$ \\
\hline & High grade & $598(54.2)$ & $283(56.8)$ & $315(52.1)$ \\
\hline & Not applicable/unknown & $368(33.4)$ & $163(32.7)$ & $205(33.9)$ \\
\hline \multirow[t]{3}{*}{ T-Stage $\S$} & $\mathrm{T} 1$ & $171(15.5)$ & $76(15.3)$ & $95(15.7)$ \\
\hline & $\mathrm{T} 2-\mathrm{T} 4$ & $516(46.8)$ & $232(46.6)$ & $284(46.9)$ \\
\hline & Other/unknown & $416(37.7)$ & $190(38.2)$ & $226(37.4)$ \\
\hline \multirow[t]{2}{*}{ Malignancy of tumor*,§ } & $\begin{array}{l}\text { Locally aggressive }+ \text { rarely meta- } \\
\text { static }\end{array}$ & $86(7.8)$ & $28(5.6)$ & $58(9.6)$ \\
\hline & Malignant & $1014(92.2)$ & $468(94.4)$ & $546(90.4)$ \\
\hline \multirow{3}{*}{$\begin{array}{l}\text { Metastasis until time of study } \\
\text { inclusion* }\end{array}$} & No metastasis & $604(54.8)$ & $252(50.6)$ & $352(58.2)$ \\
\hline & Metastasis & $365(33.1)$ & $180(36.1)$ & $185(30.6)$ \\
\hline & Unknown & $134(12.1)$ & $66(13.3)$ & $68(11.2)$ \\
\hline \multirow[t]{4}{*}{ Tumor recurrence***,\# } & No recurrence & $794(72.0)$ & $355(71.3)$ & 439 (72.6) \\
\hline & Recurrence & $278(25.2)$ & $127(25.5)$ & $151(25.0)$ \\
\hline & Suspicion & $12(1.1)$ & $7(1.4)$ & $5(0.8)$ \\
\hline & Unknown & $19(1.7)$ & $9(1.8)$ & $10(1.7)$ \\
\hline \multirow[t]{3}{*}{ Treatment intention**,\# } & Curative & $820(74.6)$ & $359(72.5)$ & $461(76.3)$ \\
\hline & Palliative & $258(49.6)$ & $128(25.9)$ & $130(21.5)$ \\
\hline & Unknown & $21(1.9)$ & $8(4.2)$ & $13(2.2)$ \\
\hline \multirow[t]{4}{*}{ Disease status\# } & Complete remission & $491(44.5)$ & $219(44.0)$ & $272(45.0)$ \\
\hline & Partial remission/stable disease & $326(29.6)$ & $148(29.7)$ & $178(29.4)$ \\
\hline & Progressive & $160(14.5)$ & $68(13.7)$ & $92(15.2)$ \\
\hline & Unknown & $126(11.4)$ & $63(12.7)$ & $63(10.4)$ \\
\hline \multirow[t]{3}{*}{ Aftercare status*,\# } & Not in aftercare & $468(42.4)$ & $231(46.4)$ & $237(39.2)$ \\
\hline & In aftercare & $620(56.2)$ & $262(52.6)$ & $358(59.2)$ \\
\hline & Unknown & $15(1.2)$ & $5(1.0)$ & $10(1.7)$ \\
\hline \multirow[t]{3}{*}{ Surgery***,\# } & No & $126(11.4)$ & $59(11.8)$ & $67(11.1)$ \\
\hline & Yes & 969 (87.9) & $435(87.3)$ & $534(88.3)$ \\
\hline & Unknown & $8(0.7)$ & $4(0.8)$ & $4(0.7)$ \\
\hline
\end{tabular}


Table 1 (continued)

\begin{tabular}{lllll}
\hline Variable & Value & All & Financial toxicity-yes & $\begin{array}{l}\text { Financial toxicity-no } \\
\mathrm{N}=605(54.9 \%)\end{array}$ \\
\hline Chemotherapy*,**,\# & No & $\mathrm{N}=1103$ (column\%) & $\mathrm{N}=498(44.7 \%)$ & $340(56.2)$ \\
& Yes & $568(51,5)$ & $228(45.8)$ & $259(42.8)$ \\
& Unknown & $523(47.4)$ & $264(53.1)$ & $6(1.0)$ \\
Radiotherapy***,\# & No & $12(1.1)$ & $6(1.2)$ & $361(59.7)$ \\
& Yes & $646(58.6)$ & $285(57.2)$ & $229(37.9)$ \\
Treatment lines\# & Unknown & $430(39.0)$ & $201(40.4)$ & $15(2.5)$ \\
& No treatment yet (1) & $27(2.4)$ & $12(2.4)$ & $17(2.8)$ \\
& One treatment line & $26(2.4)$ & $9(1.8)$ & $275(45.5)$ \\
& More than one treatment line (2) & $547(49.6)$ & $216(43.4)$ & $257(51.6)$ \\
& Unknown (3) & $39(3.5)$ & $16(3.2)$ & $290(47.9)$ \\
\hline
\end{tabular}

${ }^{*}$ Chi-square $\mathrm{p}<0.05$

** Variable not in the multivariate model

**** Model variable adapted

\#At/until time of study inclusion

$\S_{\text {At time of diagnosis }}$

Fig. 1 Comparison of financial difficulties scale to an age- and sex-adjusted norm population

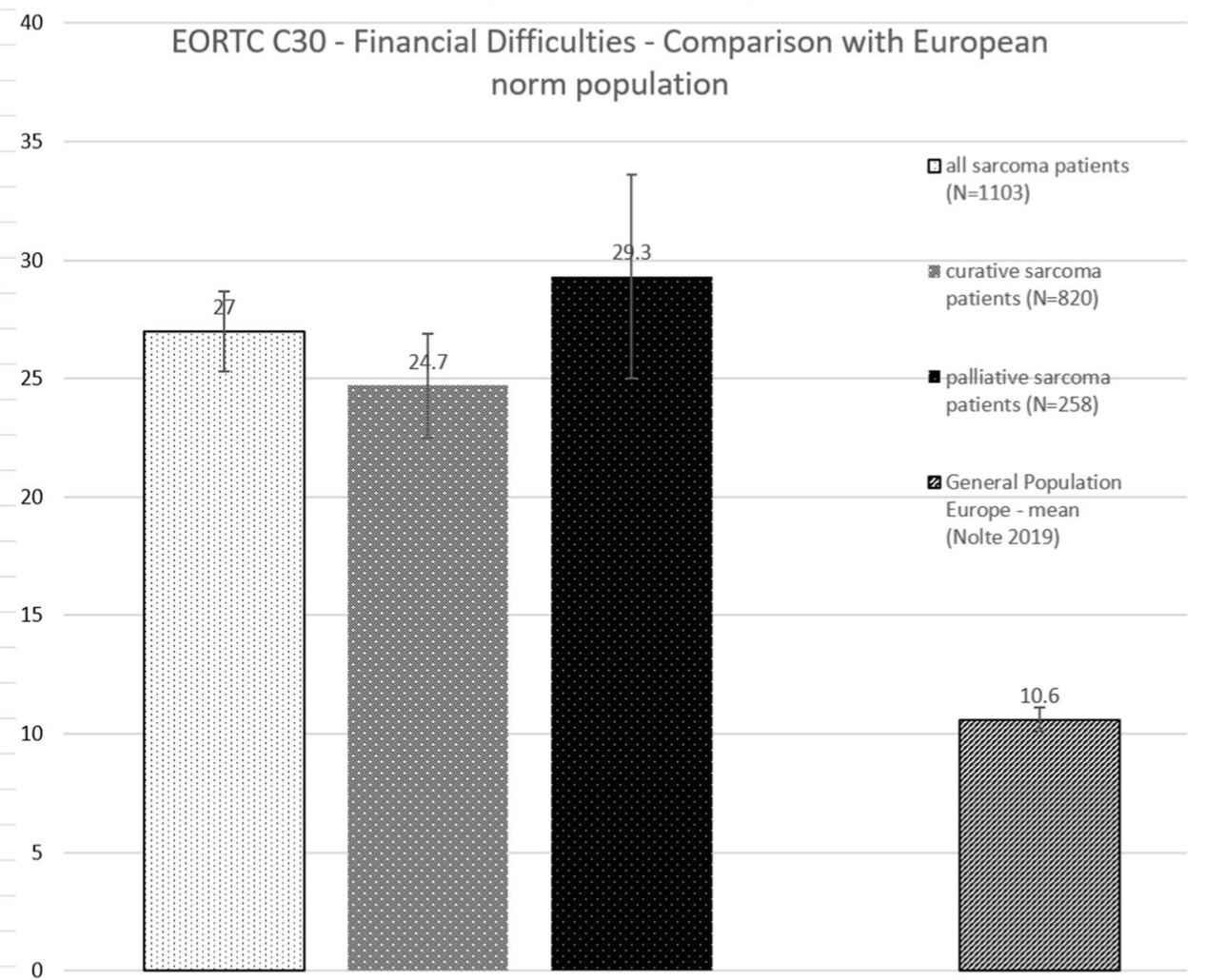

often results from OOPPs, paid by the patient for the treatment of the disease and related care and from income changes. OOPPs in sarcoma patients alone have not been evaluated and were not assessed in our study. For other cancer entities and mixed cancer populations, it has been reported that OOPPs occur at a high level in countries with higher self-payments [29] but are also present at a moderate level in countries with universal health insurance [14, 30, 31]. For a German mixed cancer population, Büttner et al. [14] found average 3-month OOPPs of $€ 205$ at the end of hospital stay, and $€ 180$ and $€ 148$ at 3 and 15 months after their hospital stay. 
Table 2 Results of the multivariate logistic regression using stepwise backward selection with having financial difficulties as the dependent variable (final model)

\begin{tabular}{|c|c|}
\hline Independent variables & OR $[95 \% \mathrm{CI}]$ \\
\hline \multicolumn{2}{|l|}{ Age at study inclusion $(\mathrm{p}=0.002)$} \\
\hline 18 to $<27.5$ years & $0.45[0.23 ; 0.88]$ \\
\hline 27.5 to $<40$ years & $0.54[0.31 ; 0.94]$ \\
\hline 40 to $<52.5$ years (reference category) & 1 \\
\hline 52.5 to $<65$ years & $0.66[0.44 ; 0.99]$ \\
\hline 65 to $<77.5$ years & $0.25[0.12 ; 0.49]$ \\
\hline$>77.5$ years & $0.27[0.12 ; 0.62]$ \\
\hline \multicolumn{2}{|l|}{ Education $(\mathrm{p}=0.023)$} \\
\hline None to secondary school (8/9 years) (ref.) & 1 \\
\hline Secondary school (10 years) & $0.57[0.39 ; 0.84]$ \\
\hline Vocational baccalaureate & $0.67[0.39 ; 1.15]$ \\
\hline High school/baccalaureate & $0.51[0.33 ; 0.79]$ \\
\hline Something else/unknown & $0.98[0.37 ; 2.60]$ \\
\hline \multicolumn{2}{|l|}{ Occupational status $(\mathrm{p}=0.081)$} \\
\hline Blue-collar worker (ref.) & 1 \\
\hline Civil servant & $1.01[0.52 ; 1.98]$ \\
\hline White collar worker & $0.95[0.64 ; 1.41]$ \\
\hline Self-employed & $1.85[1.04 ; 3.27]$ \\
\hline Unknown/not applicable & $0.76[0.41 ; 1.41]$ \\
\hline \multicolumn{2}{|l|}{ Equivalent income $(\mathrm{p}<0.001)$} \\
\hline$\leq € 1250$ (ref.) & 1 \\
\hline$€ 1250.1$ to $€ 1750$ & $0.93[0.60 ; 1.43]$ \\
\hline$€ 1750.1$ to $€ 2250$ & $0.47[0.30 ; 0.72]$ \\
\hline$€ 2250.1$ to $€ 2750$ & $0.24[0.13 ; 0.45]$ \\
\hline$\geq € 2750.1$ & $0.33[0.12 ; 0.53]$ \\
\hline Unknown & $0.58[0.35 ; 0.94]$ \\
\hline \multicolumn{2}{|l|}{ Employment status $(\mathrm{p}=0.002)$} \\
\hline Employed/self-employed (ref.) & 1 \\
\hline Unemployed & $1.58[0.75 ; 3.31]$ \\
\hline Disability pension & $3.29[1.95 ; 5.54]$ \\
\hline $\begin{array}{l}\text { Early retirement/retirement pension/partial } \\
\text { retirement }\end{array}$ & $1.13[0.61 ; 2.11]$ \\
\hline Housewife/houseman & $1.40[0.54 ; 3.62]$ \\
\hline School, apprenticeship, study & $1.24[0.38 ; 4.10]$ \\
\hline Unknown & $1.34[0.30 ; 6.02]$ \\
\hline \multicolumn{2}{|l|}{ Sick leave $(\mathrm{p}<0.001)$} \\
\hline No (ref.) & 1 \\
\hline Yes & $2.40[1.60 ; 3.62]$ \\
\hline Unknown & $1.12[0.37 ; 3.39]$ \\
\hline \multicolumn{2}{|l|}{ Disabled person pass $(\mathrm{p}<0.001)$} \\
\hline No (ref.) & 1 \\
\hline Yes & $2.52[1.81 ; 3.49]$ \\
\hline Unknown & $1.30[0.21 ; 8.03]$ \\
\hline \multicolumn{2}{|l|}{ Time since diagnosis $(0$ to $<0.5$ years $)(\mathrm{p}=0.024)$} \\
\hline 0 to $<0.5$ years (ref.) & 1 \\
\hline 0.5 to $<1$ year & $1.31[0.77 ; 2.22]$ \\
\hline 1 to $<2$ years & $0.87[0.52 ; 1.47]$ \\
\hline 2 to $<5$ years & $0.83[0.51 ; 1.36]$ \\
\hline
\end{tabular}

Table 2 (continued)

\begin{tabular}{ll}
\hline Independent variables & OR $[95 \% \mathrm{CI}]$ \\
\hline \multicolumn{1}{c}{ More than 5 years } & $0.58[0.35 ; 0.94]$ \\
Metastasis until baseline $(\mathrm{p}=0.054)$ & \\
No metastasis (ref.) & 1 \\
Metastasis & $1.38[0.97 ; 1.97]$ \\
Unknown & $1.56[1.01 ; 2.41]$ \\
Disease status $(\mathrm{p}=0.010)$ & \\
Complete remission (ref.) & 1 \\
Partial remission/stable disease & $0.96[0.67 ; 1.37]$ \\
Progressive & $0.48[0.30 ; 0.77]$ \\
Unknown & $1.11[0.68 ; 1.82]$ \\
\hline
\end{tabular}

Variables included in the full model before backward selection: sex, age at study inclusion, household size, children in household, school leaving certificate, occupational status, equivalized income, employment status, sick leave, disabled person pass, time since diagnosis, sarcoma type, site, grading, T-Stage, malignancy of tumor, metastasis until baseline, tumor recurrence, disease status, aftercare status, treatment lines, and treatment received

Income losses may also have an impact on financial difficulties in cancer patients. In our study, regression analysis showed that higher income was significantly associated with lower chances of reporting financial toxicity. This is also in line with previous studies in other cancer populations [32]. Changes in income are often related to changes in one's ability to work. Approximately $60-70 \%$ of all cancer patients return to work [15, 33], with the rest going into retirement or applying for disability pensions. Patients with sarcoma show the lowest return to work rates [34] and are considered to have a higher risk of not returning to work $[35,36]$. A study by Laros et al. [37] showed that sarcoma patients in Germany had an average unemployment rate of 8.8 months and that for $67 \%$ of the sarcoma patients, the work situation changed after the unemployment phase. Patients in Germany are allowed up to 72 weeks of sick leave before returning to work or applying for a pension. In our study, $23.3 \%$ of the patients were currently on sick leave, and sick leave was associated with a higher chance for reporting financial difficulties. Hazell et al. [38] found a similar association of sick leave and financial toxicity in lung cancer patients in the USA. Patients on sick leave in Germany receive $70 \%$ of their gross salary from their health insurance [39]. This income reduction in combination with after treatment OOPPs [14] might lead to financial problems or financial insecurity.

Another possible factor for financial difficulties during sick leave might be the reduction of income due to high surcharges not covered by the insurance [40]. If patients are not able to return to work after sick leave, it is mandatory to apply for a disability pension in Germany. The average full disability pension, which applies if you are not able to work more than $3 \mathrm{~h}$ a day, was $€ 850$ in 2019 
[41], indicating a severe income decline for patients who were fully working before their disease. Laros et al. [37] found an income loss of $62 \%$ on average for sarcoma patients in Germany who applied for a pension or partial or total unemployment benefits. The effect of this income decline is expressed in a statistically significantly increased chance of financial toxicity for patients receiving disability pension in our analysis, where having a disability pass was associated with higher odds of reporting financial toxicity. This might seem controversial because a disability pass may lead to deductions in certain services or payments (e.g., entrance fees, transportation tickets) [40]. On the other hand, patients with a disability pass might have higher OOPPs due to a need for additional services or medical aids which are not fully covered by their insurance.

Higher education was associated with lower chances of financial toxicity in our study, which is in line with findings for other cancer populations [32]. Our analysis has shown that, compared to the age group 40 to $<52.5$ years, younger and older age groups reported lower chances for reporting financial toxicity. We can only speculate as to possible reasons for this finding. Older patients may have acquired higher financial reserves during their lifetime, while our reference age group might be at a disadvantage due to financial investments, such as loans or substantial purchases. Additionally, older patients are already retired, and the income losses due to the disease may not have as great an impact because they have a stable income from their pension fund. Younger patients, on the other hand, may not yet have substantial financial investments, which might explain the lower financial toxicity reported. Furthermore, their baseline income might be lower, so that the resulting difference to the disability pension sum might be less pronounced compared to older patients. Nevertheless, studies in other cancer patients have reported that younger age is more often associated with financial toxicity [32].

Another surprising finding of our study was that patients with progressive disease had lower odds of reporting financial toxicity compared to patients with complete remission. The association of disease status and financial toxicity has been rarely assessed in the literature. Zafar et al. [42] found no statistically significant association between patients with recurrent or progressive cancer and financial distress. On the other hand, Walker et al. [43] reported more financial difficulties in patients with disease progression for advanced non-squamous NSCLC. A Finish study examining the OOPPs of breast, prostate, and colorectal cancer patients reported the lowest 6-month OOPPs for patients in remission (€224) compared to palliative patients (€603), patients with metastatic disease (€383), in rehabilitation (€264), and in primary treatment (€263) [44]. Future studies should address this issue in sarcoma patients and further evaluate possible reasons, provided that our findings can be validated in other sarcoma patient cohorts.

Patients with more than 5 years past diagnosis had lower chances of reporting financial toxicity compared to patients with $0-<0.5$ years past diagnosis. Sarcoma patients currently receiving treatment have been shown to report financial toxicity $[25,28]$. Being currently under treatment might be related to more uncertainties regarding the future where financial aspects might play an important role. Also, high OOPPs might be related to financial toxicity during treatment. Although Germany has a universal health coverage, OOPPs during treatment and in aftercare cannot be neglected [14].

A limitation of our analysis is the cross-sectional design which does not allow causal pathways to be established. Additionally, financial difficulties were assessed using a single question scale, which might not cover all aspects related to financial toxicity in cancer patients [7]. Nevertheless, the scale used is part of an internationally validated and accepted questionnaire for measuring quality of life in cancer patients. Changes in the societal financial situation might also impact financial toxicity in patients. Since data was obtained in a relatively short time frame $(09 / 2017$ to 02/2019), no significant changes in the societal financial situation occurred during that time frame which could affect the results. Finally, it is also possible that the study population is not fully representative of all sarcoma patients in Germany since participants were mostly recruited from university hospitals or specialized centers. On the other hand, we did evaluate the question of financial toxicity in one of the largest sarcoma cohorts reported in the literature, covering all relevant histological subtypes and treatment groups. With a broad set of sociodemographic and clinical variables available, we were able to identify factors which might have an impact on the chance of reporting financial difficulties that were not addressed in other studies.

\section{Conclusion}

A high proportion of sarcoma patients in Germany report financial difficulties, compared to a general norm population. This impairment appears to be relevant and needs to be addressed by the involved stakeholders, especially since many of the factors associated with financial difficulties in our study were related to income and ability to work. Both treating physicians and patients should be aware of the involved risks and the options regarding the return to a working environment should be discussed with the patients at an early stage. Additional support and consulting opportunities, taking the experience of previous patients into consideration, should be readily available. Furthermore, we believe that policy changes regarding sick leave and the disability 
pension in Germany should be considered for reducing financial inequality among sarcoma patients.

Author contribution M.Bu. wrote the article. M.E. analyzed the data. M.E., M.K.S., and L.H. developed questionnaires and study design. J.S. and M.K.S. developed the conception of the study and supervised with M.Bo. the work throughout the whole study. K.A. supervised the study from a patient's perspective. K.T. edited language. M.E., M.Bu., K.T., and S.S. developed the statistical analysis plan for this paper. S.R., P.H., B.K., D.A., and M.K.S. were responsible for the recruitment of patients or recruited patients directly. All authors have revised the manuscript critically and approved the published version. All authors have read and agreed to the published version of the manuscript.

Funding Open Access funding enabled and organized by Projekt DEAL. The PROSa study was funded by the German Cancer Aid (No. 111713).

Data Availability The data is not to be deposited but is available on request.

Code availability Code is available upon request.

\section{Declarations}

Ethics approval Ethics approval was obtained by the ethics committee of Technical University of Dresden (AZ: EK 1790422017) and the ethics committees of the participating centers.

Consent to participate Informed consent was obtained from all individual participants included in the study.

\section{Consent for publication N/A.}

Conflict of interest M.Bu. reports personal fees from Lilly and Takeda outside the submitted work.

S.S. received lecture fees from Lilly, BMS, Boehringer-Ingelheim, and Pfizer, all outside of this work.

J.S. received consulting fees from Novartis, Sanofi, A.L.K, and Lilly, all outside of this work. D.A received lecture fees from Lilly and Implantcast, all outside of this work.

M.K.S. received research funding from PharmaMar and Novartis, all outside of this work. L.H. received fees from SERVIER, outside of this work.

M.E., B.K., K.A., M.Bo., P.H., K.T., and S.R. declare that no conflicts of interest exist.

Open Access This article is licensed under a Creative Commons Attribution 4.0 International License, which permits use, sharing, adaptation, distribution and reproduction in any medium or format, as long as you give appropriate credit to the original author(s) and the source, provide a link to the Creative Commons licence, and indicate if changes were made. The images or other third party material in this article are included in the article's Creative Commons licence, unless indicated otherwise in a credit line to the material. If material is not included in the article's Creative Commons licence and your intended use is not permitted by statutory regulation or exceeds the permitted use, you will need to obtain permission directly from the copyright holder. To view a copy of this licence, visit http://creativecommons.org/licenses/by/4.0/.

\section{References}

1. van der Graaf WTA, Orbach D, Judson IR, Ferrari A (2017) Soft tissue sarcomas in adolescents and young adults: a comparison with their paediatric and adult counterparts. Lancet Oncol 18(3):e166-e175

2. Stiller CA, Trama A, Serraino D, Rossi S, Navarro C, Chirlaque MD et al (2013) Descriptive epidemiology of sarcomas in Europe: report from the RARECARE project. Eur J Cancer 49(3):684-695

3. McDonough J, Eliott J, Neuhaus S, Reid J, Butow P (2019) Health-related quality of life, psychosocial functioning, and unmet health needs in patients with sarcoma: a systematic review. Psychooncology 28(4):653-664

4. Altice CK, Banegas MP, Tucker-Seeley RD, Yabroff KR (2017) Financial hardships experienced by cancer survivors: a systematic review. J Natl Cancer Inst 109(2):djw205. https://doi.org/10.1093/ jnci/djw205

5. Azzani M, Roslani AC, Su TT (2015) The perceived cancerrelated financial hardship among patients and their families: a systematic review. Support Care Cancer 23(3):889-898

6. Gordon LG, Merollini KMD, Lowe A, Chan RJ (2017) A systematic review of financial toxicity among cancer survivors: we can't pay the co-pay. Patient 10(3):295-309

7. Witte J, Mehlis K, Surmann B, Lingnau R, Damm O, Greiner W, Winkler EC (2019) Methods for measuring financial toxicity after cancer diagnosis and treatment: a systematic review and its implications. Ann Oncol 30(7):1061-1070

8. Fenn KM, Evans SB, McCorkle R, DiGiovanna MP, Pusztai L, Sanft $\mathrm{T}$ et al (2014) Impact of financial burden of cancer on survivors' quality of life. J Oncol Pract 10(5):332-338

9. Kale HP, Carroll NV (2016) Self-reported financial burden of cancer care and its effect on physical and mental health-related quality of life among US cancer survivors. Cancer 122(8):283-289

10. Lathan CS, Cronin A, Tucker-Seeley R, Zafar SY, Ayanian JZ, Schrag D (2016) Association of financial strain with symptom burden and quality of life for patients with lung or colorectal cancer. J Clin Oncol 34(15):1732-1740

11. Zafar SY, Peppercorn JM, Schrag D, Taylor DH, Goetzinger AM, Zhong X et al (2013) The financial toxicity of cancer treatment: a pilot study assessing out-of-pocket expenses and the insured cancer patient's experience. Oncologist 18(4):381-390

12. Ramsey SD, Bansal A, Fedorenko CR, Blough DK, Overstreet KA, Shankaran V et al (2016) Financial insolvency as a risk factor for early mortality among patients with cancer. J Clin Oncol 34(9):980-986

13. Barbaret C, Brosse C, Rhondali W, Ruer M, Monsarrat L, Michaud $P$ et al (2017) Financial distress in patients with advanced cancer. PLoS One. 12(5):e0176470

14. Buttner M, Konig HH, Lobner M, Briest S, Konnopka A, Dietz A et al (2019) Out-of-pocket-payments and the financial burden of 502 cancer patients of working age in Germany: results from a longitudinal study. Support Care Cancer 27(6):2221-2228

15. Pearce A, Tomalin B, Kaambwa B, Horevoorts N, Duijts S, Mols F et al (2019) Financial toxicity is more than costs of care: the relationship between employment and financial toxicity in longterm cancer survivors. J Cancer Surviv 13(1):10-20

16. Perrone F, Jommi C, Di Maio M, Gimigliano A, Gridelli C, Pignata $S$ et al (2016) The association of financial difficulties with clinical outcomes in cancer patients: secondary analysis of 16 academic prospective clinical trials conducted in Italy. Ann Oncol 27(12):2224-2229

17. Weaver R, O'Connor M, Sobhi S, Carey Smith R, Halkett G (2020) The unmet needs of patients with sarcoma. Psychooncology 29(7):1209-1216 
18 Eichler M, Hentschel L, Richter S, Hohenberger P, Kasper B, Andreou D et al (2020) The health-related quality of life of sarcoma patients and survivors in Germany-cross-sectional results of a nationwide observational study (PROSa). Cancers (Basel) 12(12):3590. https://doi.org/10.3390/cancers 12123590

19. Hagenaars A, de Vos K, Zaidi MA (1994) Poverty statistics in the late 1980s: research based on micro-data. Office for Official Publications of the European Communities, Luxembourg

20. Aaronson NK, Ahmedzai S, Bergman B, Bullinger M, Cull A, Duez NJ et al (1993) The European Organization for Research and Treatment of Cancer QLQ-C30: a quality-of-life instrument for use in international clinical trials in oncology. J Natl Cancer Inst 85(5):365-376

21. Fayers PM, Aaronson NK, Bjordal K, Groenvold M, Curran D, Bottomley A, on behalf of the EORTC Quality of Life Group (2001) The EORTC QLQ-C30 scoring manual, 3rd edn. European Organisation for Research and Treatment of Cancer, Brussels

22. Giesinger JM, Loth FLC, Aaronson NK, Arraras JI, Caocci G, Efficace F et al (2020) Thresholds for clinical importance were established to improve interpretation of the EORTC QLQ-C30 in clinical practice and research. J Clin Epidemiol 118:1-8

23. Nolte S, Liegl G, Petersen MA, Aaronson NK, Costantini A, Fayers PM et al (2019) General population normative data for the EORTC QLQ-C30 health-related quality of life questionnaire based on 15,386 persons across 13 European countries, Canada and the Unites States. Eur J Cancer 107:153-163

24. Hudgens S, Forsythe A, Kontoudis I, D’Adamo D, Bird A, Gelderblom $H$ (2017) Evaluation of quality of life at progression in patients with soft tissue sarcoma. Sarcoma 2017:2372135

25. Paredes T, Pereira M, Moreira H, Simoes MR, Canavarro MC (2011) Quality of life of sarcoma patients from diagnosis to treatments: predictors and longitudinal trajectories. Eur J Oncol Nurs 15(5):492-499

26. Podleska LE, Kaya N, Farzaliyev F, Pottgen C, Bauer S, Taeger $\mathrm{G}$ (2017) Lower limb function and quality of life after ILP for soft-tissue sarcoma. World J Surg Oncol 15(1):84

27. Reichardt P, Leahy M, Garcia Del Muro X, Ferrari S, Martin J, Gelderblom $\mathrm{H}$ et al (2012) Quality of life and utility in patients with metastatic soft tissue and bone sarcoma: the sarcoma treatment and burden of illness in North America and Europe (SABINE) study. Sarcoma. 2012:740279

28. Srivastava A, Vischioni B, Fiore MR, Vitolo V, Fossati P, Iannalfi A et al (2013) Quality of life in patients with chordomas/chondrosarcomas during treatment with proton beam therapy. J Radiat Res 54 Suppl 1:i43-i48

29. Longo CJ, Bereza BG (2011) A comparative analysis of monthly out-of-pocket costs for patients with breast cancer as compared with other common cancers in Ontario Canada. Curr Oncol 18(1):e1-8

30. Baili P, Di Salvo F, de Lorenzo F, Maietta F, Pinto C, Rizzotto V et al (2016) Out-of-pocket costs for cancer survivors between 5 and 10 years from diagnosis: an Italian population-based study. Support Care Cancer 24(5):2225-2233

31. Céilleacher AO, Hanly P, Skally M, O'Leary E, O'Neill C, Fitzpatrick P et al (2017) Counting the cost of cancer: out-ofpocket payments made by colorectal cancer survivors. Support Care Cancer 25(9):2733-2741
32 Mols F, Tomalin B, Pearce A, Kaambwa B, Koczwara B (2020) Financial toxicity and employment status in cancer survivors. A systematic literature review. Support Care Cancer 28(12):5693-708

33. Spelten ER, Sprangers MA, Verbeek JH (2002) Factors reported to influence the return to work of cancer survivors: a literature review. Psychooncology 11(2):124-131

34. Leuteritz K, Friedrich M, Sender A, Richter D, Mehnert-Theuerkauf A, Sauter S, Geue K (2021) Return to work and employment situation of young adult cancer survivors: results from the adolescent and young adult-Leipzig study. J Adolesc Young Adult Oncol 10(2):226-233. https://doi.org/10.1089/jayao.2020.0055

35. Zambrano SC, Kollar A, Bernhard J (2020) Experiences of return to work after treatment for extremital soft tissue or bone sarcoma: between distraction and leaving the disease behind. Psychooncology 29(4):781-787

36. Kwong TN, Furtado S, Gerrand C (2014) What do we know about survivorship after treatment for extremity sarcoma? A systematic review. Eur J Surg Oncol 40(9):1109-1124

37. Laros N, Sungu-Winkler KT, Gutermuth S, Wartenberg M, Hohenberger P, Seifart U et al (2020) Career and financial situation of patients diagnosed with soft tissue sarcomas. Oncol Res Treat 43(10):539-548

38. Hazell SZ, Fu W, Hu C, Voong KR, Lee B, Peterson V et al (2020) Financial toxicity in lung cancer: an assessment of magnitude, perception, and impact on quality of life. Ann Oncol 31(1):96-102

39. https://www.bundesgesundheitsministerium.de/krankengeld.html. last Access 19.01.2021

40. Lueckmann SL, Schumann N, Hoffmann L, Roick J, Kowalski C, Dragano N et al (2020) 'It was a big monetary cut'-a qualitative study on financial toxicity analysing patients' experiences with cancer costs in Germany. Health Soc Care Community 28(3):771-780

41. Deutsche Rentenversicherung (2020) Statistik der deutschen Rentenversicherung - Rentenversicherung in Zahlen 2020

42. Zafar SY, McNeil RB, Thomas CM, Lathan CS, Ayanian JZ, Provenzale D (2015) Population-based assessment of cancer survivors' financial burden and quality of life: a prospective cohort study. J Oncol Pract 11(2):145-150

43. Walker MS, Wong W, Ravelo A, Miller PJE, Schwartzberg LS (2017) Effectiveness outcomes and health related quality of life impact of disease progression in patients with advanced nonsquamous NSCLC treated in real-world community oncology settings: results from a prospective medical record registry study. Health Qual Life Outcomes 15(1):160

44. Koskinen JP, Farkkila N, Sintonen H, Saarto T, Taari K, Roine RP (2019) The association of financial difficulties and out-of-pocket payments with health-related quality of life among breast, prostate and colorectal cancer patients. Acta Oncol 58(7):1062-1068

Publisher's note Springer Nature remains neutral with regard to jurisdictional claims in published maps and institutional affiliations. 